\title{
When are enriched strong monads double exponential monads?
}

\author{
Christopher Townsend
}

\begin{abstract}
Some categorical conditions are given that are sufficient to show that an enriched monad with a strength is a double exponential monad. The conditions hold for the double power locale monad (enriched over posets) and so as an application it is shown that the double power locale monad is a double exponential monad. A benefit is that this result about the double power locale monad can be established without the need for any detailed discussion of frame presentations or topos theory.
\end{abstract}

\section{Introduction}

The category of locales provides an example of a category where exponentials do not always exist (not all locales are locally compact) but for which double exponentiation at the Sierpiński locale does always exist; the double exponential is given by the double power locale construction, [VT04]. This example motivates a broader question: what categorical conditions can we think of that establish that a monad is a double exponential monad, in the absence of an assumption of cartesian closedness on the ambient category? The double power locale monad has a strength and the category of locales is enriched over posets and has categorical tensors that are stable under finite product. Further, the proof that the double power locale functor is a double exponential seems, at the very least, to require this level of assumptions on the ambient category $\mathcal{C}$ and the structure of the monad. So the question becomes: when are enriched strong monads double exponential monads? This paper provides some categorical conditions that

Received by the editors in April 2015 - In revised form in February 2016.

Communicated by E. Colebunders.

2010 Mathematics Subject Classification : 06D22, 18D, 18 D20.

Key words and phrases : categorical double exponential, frame, locale. 
answer the question. We show that for any strong order enriched monad $\mathbb{T}$ we have that $\mathbb{T}$ is a double exponential monad $X \mapsto A^{A^{X}}$ provided (i) $A$ is a $\mathbb{T}$-algebra, (ii) $A^{X}$ exists weakly and is given by categorical tensor; and, (iii) the contravariant functor $\mathcal{C}\left({ }_{-}, A\right)$ is fully faithful.

The structure of the paper is as follows. In the next section we recall some results about categorical tensors, focusing on how when tensors exist they induce a monad on the category $\mathcal{V}$ over which our ambient category $\mathcal{C}$ is enriched. The following section discusses weak exponentials, and describes a condition for when weak exponentials are given by tensor. The next section proves the main result, showing that the categorical conditions just outlined are sufficient for a strong monad to be double exponential. The second to last section outlines how the result can be applied to prove that the double power locale monad is a double exponential monad. The last section provides a discussion on potential further work.

\section{Order enriched categorical definitions and initial lemmas on tensors}

Let $\mathcal{C}$ be a category enriched over another category $\mathcal{V}$. We assume that $\mathcal{V}$ is a category with finite products and has 1 generating; that is, for any two morphisms $c, d: D \Longrightarrow E$ if $c a=d a$ for all $a: 1 \longrightarrow D$ then $c=d$. Any enrichment over $\mathcal{V}$ uses finite products in $\mathcal{V}$ as the monoidal structure. Consult B2.1 of [J02] for material on enriched categories; in particular we follow the notation $|\mathcal{C}|$ for the underlying ordinary category of an enriched category $\mathcal{C}$.

The definition of tensor for an object $D$ of $\mathcal{V}$ and an object $X$ of $\mathcal{C}$, is an object $D \otimes X$ of $\mathcal{C}$ together with a map $i_{X}^{D}: D \longrightarrow \mathcal{C}(X, D \otimes X)$ such that for every $Y$ and every morphism $l: D \longrightarrow \mathcal{C}(X, Y)$ there is a unique map $x_{l}: D \otimes X \longrightarrow Y$ such that $l$ factors as $D \stackrel{i_{X}^{D}}{\longrightarrow} \mathcal{C}(X, D \otimes X) \stackrel{x_{l} \circ(-)}{\longrightarrow} \mathcal{C}(X, Y)$. Put another way, (_) $\otimes X$ : $\mathcal{V} \longrightarrow|\mathcal{C}|$ is left adjoint to $\mathcal{C}\left(X,_{-}\right):|\mathcal{C}| \longrightarrow \mathcal{V}$. We use the notation $e v_{X, Y}$ for the map $x_{I d_{\mathcal{C}(X, Y)}}: \mathcal{C}(X, Y) \otimes X \longrightarrow Y$; i.e. the mate of the identity on $\mathcal{C}(X, Y)$. Below we will have a fixed object $A$ as part of our assumptions; $D_{X}$ will be used as notation for $\mathcal{C}(X, A)$ and $e v_{X}$ for $e v_{X, A}: D_{X} \otimes X \longrightarrow A$.

Say $\mathcal{C}$ has finite products and tensors. Then for any pair $X_{1}$ and $X_{2}$ of objects of $\mathcal{C}$ there is a canonical map $\Psi_{X_{1}, X_{2}}: D \otimes\left(X_{1} \times X_{2}\right) \longrightarrow\left(D \otimes X_{1}\right) \times X_{2}$ given by $x_{l_{\times}}$where $l_{\times}$is the map

$$
D \stackrel{i_{X_{1}}^{D}}{\longrightarrow} \mathcal{C}\left(X_{1},\left(D \otimes X_{1}\right)\right) \stackrel{(-) \times I d_{X_{2}}}{\longrightarrow} \mathcal{C}\left(X_{1} \times X_{2},\left(D \otimes X_{1}\right) \times X_{2}\right)
$$

Tensors are said to be stable under finite products provided this canonical map is always an isomorphism. Note that the condition is equivalent to the same condition restricted to $X_{1}=1$. Notice that the mate of

$$
D \stackrel{i_{1}^{D}}{\longrightarrow} \mathcal{C}(1, D \otimes 1) \stackrel{(-) \times I d_{X}}{\longrightarrow} \mathcal{C}(X,[D \otimes 1] \times X)
$$

is the same as the mate of

$$
D \stackrel{i_{X}^{D}}{\longrightarrow} \mathcal{C}(X, D \otimes X) \stackrel{\Psi_{1, X^{\circ}(-)}^{\longrightarrow}}{\longrightarrow} \mathcal{C}(X,[D \otimes 1] \times X)
$$


by definition of $\Psi$. It follows that if tensors are stable under product then the diagram

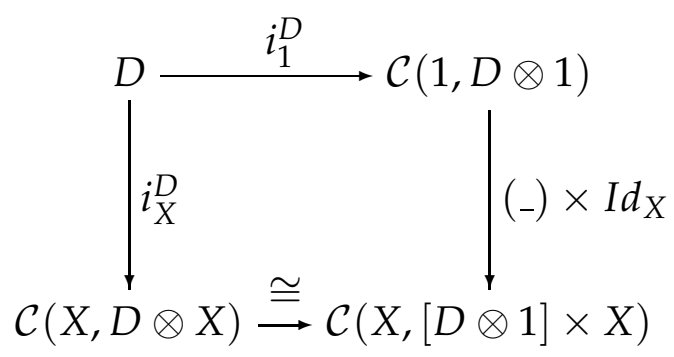

commutes. Therefore every arrow $X \longrightarrow D \otimes X$ in the image of $i_{X}^{D}$ can be expressed as $\left(a, I d_{X}\right): X \longrightarrow D \otimes X$ for some $a: 1 \longrightarrow D \otimes 1$. This will be exploited when we come to looking at natural transformations between functors of the form $\mathcal{C}\left({ }_{-} \times X, A\right):\left|\mathcal{C}^{o p}\right| \longrightarrow \mathcal{V}$.

Notice also that the following diagram commutes, provided tensors are stable under product:

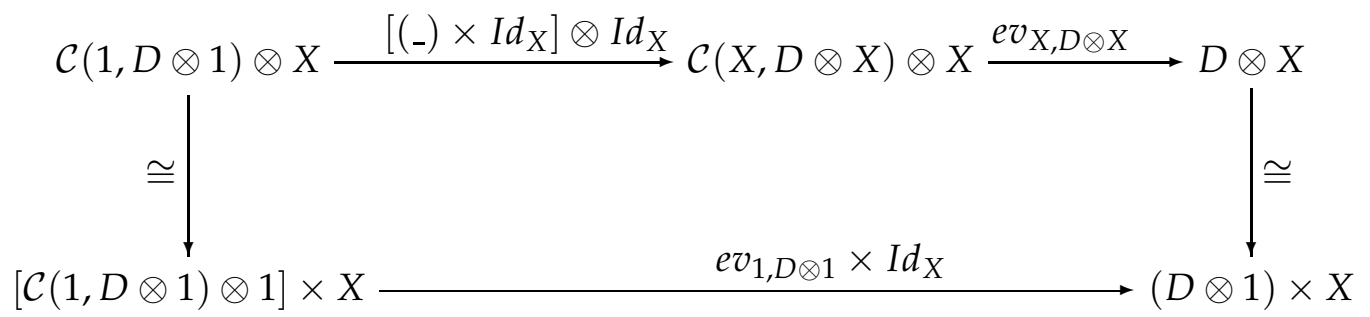

This follows by unwinding the definitions of the various isomorphisms involved. By definition of tensor, for each object $X$ of $\mathcal{C}$, a monad $\mathbb{K}_{X}$ is induced on $\mathcal{V}$ whose functor part is given by $D \mapsto \mathcal{C}(X, D \otimes X)$; this is the monad on $\mathcal{V}$ induced by the adjunction (_) $\otimes X \dashv \mathcal{C}\left(X_{-}\right)$. The last two diagrams show that provided tensors are stable under product, $\left(_{-}\right) \times I d_{X}$ induces a monad morphism from $\mathbb{K}\left(=\mathbb{K}_{1}\right)$ to $\mathbb{K}_{X}$. It follows that for any $X$ and $Y, \mathcal{C}(X, Y)$ is a $\mathbb{K}$ algebra; its structure map is given by $\mathcal{C}(1, \mathcal{C}(X, Y) \otimes 1) \stackrel{(-) \times I d_{X}}{\longrightarrow} \mathcal{C}(X, \mathcal{C}(X, Y) \otimes X) \stackrel{e v_{X, Y} \circ(-)}{\longrightarrow} \mathcal{C}(X, Y)$

Example 2.1. The category of sets, enriched over itself, is an example: $X \otimes Y$ is given by $X \times Y$, from which it is clear that the tensor is stable under finite products.

Example 2.2. The category of locales, Loc, is enriched over Pos, the category of posets. It has tensors that are stable under products. For any poset $P$, monotone maps $P \longrightarrow \operatorname{Loc}(X, Y)$ are in order preserving bijection with $\operatorname{Loc}(\operatorname{Idl}(P) \times X, Y)$, where $\operatorname{Idl}(P)$ is the locale whose frame of opens is the set of upper closed subsets of $P$. In other words $P \otimes X$ exists and is given by $\operatorname{Idl}(P) \times X$, from which it is clear that the tensor is stable under products. The monad induced on Pos is the ideal completion monad (its functor part sends each poset to its set of ideals; that is, lower closed and directed subsets). The category of algebras is therefore the category $\mathbf{d c p o}$, of directed complete posets.

With these basic facts about tensor recalled and examples given, we can now progress with an initial lemma. 
Lemma 2.3. Assume that $\mathcal{C}$, enriched over $\mathcal{V}$, has finite products and tensors that are stable under finite product. Then,

(a) for any objects $X, Y$ and $A$ of $\mathcal{C}$, given any natural transformation $\alpha: \mathcal{C}\left({ }_{-} \times X, A\right) \longrightarrow \mathcal{C}\left(\_\times Y, A\right)$,

(i) $\alpha_{D_{X} \otimes 1}\left(e v_{X}\right): D_{X} \otimes Y \longrightarrow A$ is equal to

$$
D_{X} \otimes Y \stackrel{\alpha_{1} \otimes I d_{Y}}{\longrightarrow} D_{Y} \otimes Y \stackrel{e v_{Y}}{\longrightarrow} A ; \text { and }
$$

(ii) $\alpha_{1}$ is a $\mathbb{K}$-algebra homomorphism.

(b) For any two natural transformations $\alpha, \beta: \mathcal{C}\left({ }_{-} \times X, A\right) \longrightarrow \mathcal{C}\left({ }_{-} \times Y, A\right)$, $\alpha_{1}=\beta_{1}$ if and only if $\alpha_{D_{X} \otimes 1}\left(e v_{X}\right)=\beta_{D_{X} \otimes 1}\left(e v_{X}\right)$.

Part (a)(ii) tells us that natural transformations $\operatorname{Loc}(-\times X, A)$ $\operatorname{Loc}\left(\_\times Y, A\right)$ give rise to dcpo homomorphisms. Part (b) will be key to the proof of the main result.

Proof. (a)(i). By definition of tensor, a proof is needed that

$$
D_{X} \stackrel{i_{Y}^{D_{X}}}{\longrightarrow} \mathcal{C}\left(Y, D_{X} \otimes Y\right) \stackrel{\alpha_{D_{X} \otimes 1}\left(e v_{X}\right) \circ(-)}{\longrightarrow} \mathcal{C}(Y, A)
$$

is equal to

$$
D_{X} \stackrel{\alpha_{1}}{\longrightarrow} D_{Y}
$$

Since 1 is generating, and every $a: 1 \longrightarrow D_{X}$ factors as $\left(i_{1}^{D_{X}}(a)\right)\left(e v_{X}\right)$, the two arrows are equal because $\alpha$ is natural at each $i_{1}^{D_{X}}(a): 1 \longrightarrow D_{X} \otimes 1$ and, as observed above, $i_{Y}^{D_{X}}(a)$ factors as $Y \stackrel{\left(i_{1}^{D_{X}}(a), I d_{Y}\right)}{\longrightarrow}\left(D_{X} \otimes 1\right) \times Y$

(a)(ii). We must check for any $\phi: 1 \longrightarrow \mathcal{C}(X, A) \otimes 1$, that

$$
\alpha_{1}\left(e v_{X}\left(\phi \times I d_{X}\right)\right)=e v_{Y}\left(\left(\alpha_{1} \otimes 1\right) \phi \times I d_{Y}\right)
$$

The right hand side is equal to $e v_{Y}\left(\alpha_{1} \otimes I d_{Y}\right)\left(\phi \times I d_{Y}\right)$ and by (a)(i) this is equal to $\alpha_{D_{X} \otimes 1}\left(e v_{X}\right)\left(\phi \times I d_{X}\right)$ and so this stage of the proof follows by naturality at $\phi$.

One way round for (b) follows from (a)(i). For the other way round observe that for any natural transformation $\gamma: \mathcal{C}\left({ }_{-} \times X, A\right) \longrightarrow \mathcal{C}\left({ }_{-} \times Y, A\right)$ and for any $a: 1 \longrightarrow D_{X}$, it is clear from naturality that $\gamma_{1} a=\left[\gamma_{D_{X} \otimes 1}\left(e v_{A}\right)\right]\left(a \times I d_{Y}\right)$.

\section{Weak exponentials as tensors}

If $X$ and $A$ are two objects of a category $\mathcal{C}$, then a weak exponential $W^{[X, A]}$ is an object of $\mathcal{C}$ together with a map wev $X, A: W^{[X, A]} \times X \longrightarrow A$ such that for every map $a: Z \times X \longrightarrow A$ there is a map $f_{a}: Z \longrightarrow W^{[X, A]}$ such that $a$ factors as $w e v_{X, A}\left(f_{a} \times I d_{X}\right)$. In other words a weak exponential is the same thing as an exponential but without the uniqueness requirement placed on $f_{a}$. For interest, 
note that if the definition of weak exponential was weakened to require the existence of $f_{a}$ only in the case $Z=1$, then $D_{X} \otimes 1$ would always be a weak exponential $W^{[X, A]}$.

Weak exponentials are important in our context because in their presence the natural transformations that are of interest to us are uniquely determined by their actions on the weak evaluation map wev:

Lemma 3.1. If $\mathcal{C}$ is a category, with finite products, enriched over $\mathcal{V}$ and $A$ an object of $\mathcal{C}$ such that $W^{[X, A]}$ exists for every $X$, then every natural transformation $\mathcal{C}\left({ }_{-} \times X, A\right) \longrightarrow \mathcal{C}\left({ }_{-} \times Y, A\right)$ is uniquely determined by $\alpha_{W[X, A]}\left(\right.$ wev $\left._{X, A}\right)$

Proof. This is immediate from naturality and the definition of weak exponential (and our assumption that 1 generates $\mathcal{V}$ ) because every $a: Z \times X \longrightarrow A$ factors as $w_{e v} v_{X, A}\left(f_{a} \times I d_{X}\right)$.

If $\mathcal{C}$ has tensors that are stable under products then we say that the weak exponential $W^{[X, A]}$, when it exists, is given by the tensor $D_{X} \otimes 1$ provided the map $\left(D_{X} \otimes 1\right) \times X \stackrel{\cong}{\longrightarrow} D_{X} \otimes X \stackrel{e v_{X}}{\longrightarrow} A$ makes $D_{X} \otimes 1$ into a weak exponential. The next lemma provides some insight into the relationships between the various categorical statements that we are discussing:

Lemma 3.2. Let $\mathcal{C}$ be a category over $\mathcal{V}$ with finite products and tensors that are stable under product, and $X$ and $A$ two objects of $\mathcal{C}$. Then $\mathcal{C}$ has weak exponentials $W^{[X, A]}$, given by the tensor $D_{X} \otimes 1$, if and only if for every object $Z$ there exists a map $r_{Z}: D_{Z \times X} \otimes Z \longrightarrow D_{X} \otimes 1$ such that $e v_{X}\left(r_{Z} \times I d_{X}\right)=e v_{Z \times X}$.

Proof. Say $D_{X} \otimes 1$ is a weak exponential, then the $r_{Z}$ required exists for any $Z$, by applying the definition of weak exponential to $e v_{Z \times X}:\left(D_{Z \times X} \otimes Z\right) \times X \longrightarrow A$.

In the other direction, say we are given $a: Z \times X \longrightarrow A$, then $a$ must factor as $e v_{Z \times X}\left(p_{a}, I d_{Z \times X}\right)$ for some $p_{a}: 1 \longrightarrow D_{Z \times X} \otimes 1$. But then $r_{Z}\left(p_{a}, I d_{Z}\right)$ is the morphism required to prove that $D_{X} \otimes 1$ is a weak exponential.

It is well know that the category of locales has weak exponentials, $W^{[X, S]}$, given by tensor, $\operatorname{Idl}\left(D_{X}\right)$, where we are taking $A=\mathrm{S}$, the Sierpinski locale, for $\mathcal{C}=$ Loc (and so for any locale $X, D_{X} \cong \mathcal{O} X$, the opens of $X$ ). For example you can exploit the facts that $\operatorname{Idl}\left(D_{X}\right) \cong S^{\operatorname{Spec}\left(D_{X}\right)}$ and $S$ is injective. The lemma can also be applied; since the opens of $Z \times X$ are in order preserving bijection with suplattice homomorphisms $\mathcal{O X} \longrightarrow \mathcal{O} Z^{o p}$ there is a forgetful monotone map from $D_{Z \times X} \longrightarrow \operatorname{Loc}\left(Z, \operatorname{Idl}\left(D_{X}\right)\right)$, which defines a map $r_{Z}: \operatorname{Idl}\left(D_{Z \times X}\right) \times$ $Z \longrightarrow \operatorname{Idl}\left(D_{X}\right)$ for any locale $Z$.

\section{Main result}

Before we state and prove our main result, we must be clearer about what is meant by a double exponential monad. For objects $X$ and $A$ in $\mathcal{C}, A^{X}$ does not necessarily exist in $\mathcal{C}$ as we are not making the assumption that $\mathcal{C}$ is cartesian closed. However, the presheaf $\mathcal{C}(-\times X, A):\left|\mathcal{C}^{o p}\right| \longrightarrow \mathcal{V}$ is the exponential 
$\mathcal{C}\left({ }_{-}, A\right)^{\mathcal{C}\left({ }_{-}, X\right)}$ in the presheaf category $\left[\left|\mathcal{C}^{o p}\right|, \mathcal{V}\right]$. So we can define the double exponential $A^{A^{X}}$ to be an object of $\mathcal{C}$ with the property that morphisms $p: Y \longrightarrow A^{A^{X}}$ are (naturally in $Y$ ) in bijection with natural transformations from $\mathcal{C}\left(\_\times X, A\right)$ to $\mathcal{C}\left({ }_{-} \times Y, A\right)$. An enriched monad $\mathbb{T}=(T, \eta, \mu)$ on an enriched category $\mathcal{C}$ is a double exponential monad with respect to an object $A$ provided TX is a double exponential $A^{A^{X}}$, naturally in $X$, and under the bijections that this establishes the unit $\eta_{X}$ is mapped to the identity natural transformation on $\mathcal{C}(-\times X, A)$ and the natural transformation $\mathcal{C}\left({ }_{-}, \mu_{X}\right)$ is $\mathcal{C}\left({ }_{-}, A\right)^{\bigotimes^{X}}$, where $\otimes^{X}: \mathcal{C}\left({ }_{-} \times X, A\right) \longrightarrow$ $\mathcal{C}(-\times T X, A)$ is the mate of the identity on $T X$.

If $\mathbb{T}$ is a double exponential monad, then a strength can be defined on it by defining, for any $Z$ and $X$ of $\mathcal{C}, t_{Z, X}: Z \times T X \longrightarrow T(Z \times X)$ to be the map corresponding to the natural transformation $\mathcal{C}(-\times Z \times X, A) \stackrel{\otimes_{(-) \times Z}^{X}}{\longrightarrow}$ $\mathcal{C}\left(\_\times Z \times T X, A\right)$. If $\mathbb{T}$ is a monad with a strength then it is a double exponential monad provided that also the strength is, up to isomorphism, that determined by the double exponential structure (i.e. determined by $\bigotimes_{(-) \times Z}^{X}$ ).

Theorem 4.1. Let $\mathcal{V}$ be a finite product category with 1 generating and $\mathcal{C}$ a category enriched over $\mathcal{V}$ with tensors that are stable under product. Denote by $\mathbb{K}$ the monad on $\mathcal{V}$ induced by the assumption that $\mathcal{C}$ has tensors (i.e. its functor part is $D \mapsto \mathcal{C}(1, D \otimes 1)$ ).

Let $A$ be an object of $\mathcal{C}$ such that for any object $X$ of $\mathcal{C}$ the weak exponential $W^{[X, A]}$ exists and is given by tensor. Then for any strong $\mathcal{V}$-monad $\mathbb{T}$ on $\mathcal{C}$, we have that $\mathbb{T}$ is isomorphic to the double exponential monad induced by A provided

(i) $A$ is a $\mathbb{T}$-algebra; and,

(ii) the functor $U_{A}:\left|\mathcal{C}_{\mathbb{T}}\right| \longrightarrow\left(\mathcal{V}^{\mathbb{K}}\right)^{\text {op }}$ given by $U_{A}(X)=\mathcal{C}(X, A)$ is full and faithful.

For clarity we note that the functor $U_{A}$ is a contravariant functor from the underlying ordinary category of the Kleisli category determined by $\mathbb{T}$ to the category of algebras on $\mathcal{V}$ induced by the tensor. It is given by

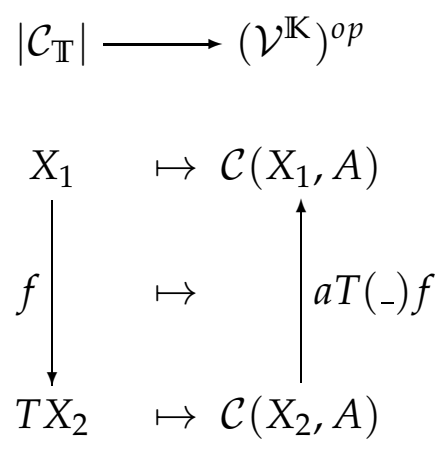

where $a: T A \longrightarrow A$ is the structure map on $A$. That this is well defined is clear from earlier lemmas (to see that $a T\left(_{-}\right) f$ is a $\mathbb{K}$ algebra homomorphism, observe that $\alpha_{Z}\left(Z \times X_{2} \stackrel{c}{\longrightarrow} A\right)=Z \times X_{1} \stackrel{I d_{Z} \times f}{\longrightarrow} Z \times T X_{2} \stackrel{t_{Z, X_{2}}}{\longrightarrow} T\left(Z \times X_{2}\right) \stackrel{T c}{\longrightarrow} T A \stackrel{a}{\longrightarrow} A$ determines a natural transformation with $\alpha_{1}=a T(-) f$ and apply Lemma 2.3 (a)(ii)). 
Proof. We start with a proof that for any objects $X$ and $Y$ of $\mathcal{C}$, morphisms $p: Y \longrightarrow T X$ are in bijection with the collection of natural transformations from $\mathcal{C}(-\times X, A)$ to $\mathcal{C}(-\times Y, A)$.

For every object $X$ of $\mathcal{C}$, consider the functor $F_{X}:|\mathcal{C}| \longrightarrow\left|\mathcal{C}_{\mathbb{T}}\right|$ that sends $Z$ to $Z \times X$ on objects and sends a morphism $f: Z_{1} \longrightarrow Z_{2}$ to $Z_{1} \times X \stackrel{f \times I d_{X}}{\longrightarrow}$ $Z_{2} \times X \stackrel{\eta Z_{2} \times X}{\longrightarrow} T\left(Z_{2} \times X\right)$. Clearly $\mathcal{C}(-\times X, A)=U_{A} F_{X}$.

Given $p: Y \longrightarrow T X$, define $\alpha^{p}: F_{Y} \longrightarrow F_{X}$ by defining $\alpha_{Z}^{p}$ to be the composite

$$
Z \times Y \stackrel{I d_{Z} \times p}{\longrightarrow} Z \times T X \stackrel{t_{Z, X}}{\longrightarrow} T(Z \times X),
$$

where $t$ is the strength on $\mathbb{T}$. By exploiting the fact that the strength $t_{Z, X}$ is natural in $Z$, it can be checked that $\alpha^{p}$ is a natural transformation and so gives rise to a natural transformation $\beta^{p} \equiv U_{A}\left(\alpha^{p}\right)$ from $\mathcal{C}(-\times X, A)$ to $\mathcal{C}(-\times Y, A)$. By exploiting the fact that $t_{1, X}$ is (canonically isomorphic to) the identity on $T X$ (by definition of strength) we see that $\alpha_{1}^{p}=p$.

On the other hand given a natural transformation $\beta$ from $\mathcal{C}(-\times X, A)$ to $\mathcal{C}(-\times Y, A)$, we know by part (a)(ii) of Lemma 2.3 that $\beta_{1}$ is a $\mathbb{K}$-algebra homomorphism and so by assumption there is some $p_{\beta}: Y \longrightarrow T X$ such that $U_{A}\left(p_{\beta}\right)=\beta_{1}$. By combining (b) of Lemma 2.3 and Lemma 3.1 we know that $\beta$ is uniquely determined by $\beta_{1}$ and so as we have assumed that $U_{A}$ is faithful, a bijection is established between morphisms $Y \longrightarrow T X$ and natural transformations $\mathcal{C}(-\times X, A)$ to $\mathcal{C}(-\times Y, A)$. For clarity we note that given $p: Y \longrightarrow T X$, then the corresponding natural transformation, $\beta^{p}$, sends any $b: Z \times X \longrightarrow A$ to

$$
Z \times Y \stackrel{I d_{Z} \times p}{\longrightarrow} Z \times T X \stackrel{t_{Z, X}}{\longrightarrow} T(Z \times X) \stackrel{T b}{\longrightarrow} T A \stackrel{a}{\longrightarrow} A .
$$

It is then clear that the bijection is natural in $Y$ and further that the mate of $\eta_{X}: X \longrightarrow T X$ must be the identity natural transformation, this last by exploiting the fact that $\eta_{Z \times X}$ must factor as $t_{Z, X}\left(I d_{Z} \times \eta_{X}\right)$ for any $Z$, by definition of strength. To see that the bijection is natural in $X$, say we are given $g: X_{1} \longrightarrow X_{2}$, then it must be checked for any $p: Y \longrightarrow T X_{1}$ and any $b: Z \times X_{2} \longrightarrow A$ that

$$
Z \times Y \stackrel{I d_{Z} \times p}{\longrightarrow} Z \times T X_{1} \stackrel{I d_{Z} \times T g}{\longrightarrow} Z \times T X_{2} \stackrel{t_{Z, X_{2}}}{\longrightarrow} T\left(Z \times X_{2}\right) \stackrel{T b}{\longrightarrow} T A \stackrel{a}{\longrightarrow} A
$$

is equal to

$$
Z \times Y \stackrel{I d_{Z} \times p}{\longrightarrow} Z \times T X_{1} \stackrel{t_{Z, X_{1}}}{\longrightarrow} T\left(Z \times X_{1}\right) \stackrel{T\left(I d_{X} \times g\right)}{\longrightarrow} T\left(Z \times X_{2}\right) \stackrel{T b}{\longrightarrow} T A \stackrel{a}{\longrightarrow} A
$$

which follows by naturality of $t_{Z, X}$ at $X$.

We also must have that $\mathcal{C}\left({ }_{-}, \mu_{X}\right)$ is $\mathcal{C}\left({ }_{-}, A\right)^{\otimes^{X}}$, up to the bijections established. To see this it can be checked that for any $p: Y \longrightarrow T T X$ that $\beta^{\mu_{X} p}=\beta^{p} \beta^{I d_{T X}}$, i.e. for any $b: Z \times X \longrightarrow A$ that

$$
Z \times Y \stackrel{I d_{Z} \times p}{\longrightarrow} Z \times T T X \stackrel{I d_{Z} \times \mu_{X}}{\longrightarrow} Z \times T X \stackrel{t_{Z, X}}{\longrightarrow} T(Z \times X) \stackrel{T b}{\longrightarrow} T A \stackrel{a}{\longrightarrow} A
$$

is equal to 


$$
\begin{array}{r}
Z \times Y \stackrel{I d_{Z} \times p}{\longrightarrow} Z \times T T X \stackrel{t_{Z, T X}}{\longrightarrow} T(Z \times T X) \stackrel{T\left(I d_{Z} \times I d_{T X}\right)}{\longrightarrow} T(Z \times T X) \stackrel{T t_{Z, X}}{\longrightarrow} \\
T T(Z \times X) \stackrel{T T b}{\longrightarrow} T T A \stackrel{T a}{\longrightarrow} T A \stackrel{a}{\longrightarrow} A .
\end{array}
$$

This can be seen by exploiting the facts that $a T a=a \mu_{A}, \mu$ is natural (at $b$ ) and that $\mu_{Z \times X}\left(T t_{Z, X}\right) t_{Z, T X}=t_{Z, X}\left(I d_{Z} \times \mu_{X}\right)$, by definition of strength.

Finally, the strength $t$ of $\mathbb{T}$ must be shown to be that induced by the double exponential structure. Say we are given $b: Z_{1} \times Z \times X \longrightarrow A$, then it must be checked that

$Z_{1} \times Z \times T X \stackrel{I d_{Z_{1}} \times t_{Z, X}}{\longrightarrow} Z_{1} \times T(Z \times X) \stackrel{t_{Z_{1}, Z \times X}}{\longrightarrow} T\left(Z_{1} \times Z \times X\right) \stackrel{T b}{\longrightarrow} T A \stackrel{a}{\longrightarrow} A$

is equal to

$$
Z_{1} \times Z \times T X \stackrel{t_{Z_{1} \times Z, X}}{\longrightarrow} T\left(Z_{1} \times Z \times X\right) \stackrel{T b}{\longrightarrow} T A \stackrel{a}{\longrightarrow} A
$$

which is clear from the definition of strength.

\section{Application}

The double power locale monad, $\mathbb{P}$, was initially defined as the composite, in either order, of the lower and the upper power locale monad, [JV91]. It does not matter which order is taken because the upper and lower power locale monads commute with each other. If $X$ is a locale then the frame of opens of the double power locale $\mathbb{P X}$, is given by the free frame on $\mathcal{O} X$, keeping the dcpo structure on $\mathcal{O} X$ fixed. Notice then that $S \cong \mathbb{P} 0$ because the frame of opens of $S$ is the free frame on the singleton set 1 (and the frame of opens of the zero locale is the singleton set). So $\mathrm{S}$ is a $\mathbb{P}$-algebra.

Both the lower and upper power locale monads have strengths; this can be seen by exploiting the fact that locale product can be given by either suplattice or preframe tensor. Therefore the double power locale monad has a strength as it is easy to check that the composition of any two commuting monads, both with a strength, has a strength. It is clear that $U_{\mathbb{S}}: \mathbf{L o c}_{\mathbb{P}} \longrightarrow \mathbf{P o s}^{o p}$ is the functor that sends a morphism $Y \longrightarrow \mathbb{P X}$ to its corresponding dcpo homomorphism; it is therefore faithful. Equally any dcpo homomorphism $\mathcal{O X} \longrightarrow \mathcal{O Y}$ arises in this way and so we have checked all the conditions of our main theorem and can conclude that $\mathbb{P}$ is a double exponential monad.

\section{Discussion}

That the double power locale monad is a double exponential monad was originally shown in [VT04]. The proof offered here, it is hoped, provides some insight into categorical techniques that can be deployed to obtain the result. Trivially the main result can be applied to cartesian closed categories, so the challenge of 
finding non-trivial applications is finding categories that are not cartesian closed but for which double exponentiation does exist (for some object $A$ ). Aside from Loc the author has been unable to think of any; the relationship between the monad induced on the category $\mathcal{V}$ and the points of the double power locale monad seems to be quite particular. To take these ideas further one instinct is to tamper with Pos; for example, the points of the lower and upper power locales are natural transformations between functors Loc $^{o p} \longrightarrow$ SLat, where SLat is the category of semilattices. But this fails then to be double exponentiation because the Yoneda lemma does not embed Loc into $\left[\mathbf{L o c}^{o p}, \mathbf{S L a t}\right]$. On the other hand if the situation is unique, perhaps it is characterizing Loc? Indeed the category of locales is the opposite of the category of order internal distributive lattices in the category dcpo; is it possible for a category to be the category of internal distributive lattices on the category of algebras of its enrichment (and to have double exponentiation) without this somehow forcing it to be Loc? The challenge in this idea seems to be in proving that the enrichment is necessarily Pos.

Another avenue is to see whether the categorical techniques offered here can be deployed to provide a result about indexed categories, with $\mathcal{V}$ the base category. This idea is plausible because the double power locale monad is double exponentiation relative to any topos $\mathcal{E}$, but a direct application of the result offered here is not possible because 1 is not generating in the category of posets relative to an arbitrary topos $\mathcal{E}$. The author hopes to make an indexed version of our main result the topic of a follow up paper.

\subsection{Acknowledgement}

This paper is dedicated to my daughter, Hannah Townsend.

\section{References}

[J02] Johnstone, P.T. Sketches of an elephant: A topos theory compendium. Vols 1, 2, Oxford Logic Guides 43, 44, Oxford Science Publications, 2002.

[JV91] Johnstone, P.T., and Vickers, S.J. "Preframe presentations present", in Carboni, Pedicchio and Rosolini (eds) Category Theory - Proceedings, Como, 1990 (Springer Lecture Notes in Mathematics 1488, 1991), 193-212.

[VT04] Vickers, S.J. and Townsend, C.F. A Universal Characterization of the Double Power Locale. Theoretical Computer Science 316 (2004) 297-321.

8, Gordon Villas, Aylesbury Road, Tring, HERTS HP23 4DJ, U.K.

Email: info@christophertownsend.org 